Check for updates

Cite this: J. Mater. Chem. B, 2020, 8, 4202

Received 3rd January 2020, Accepted 13th March 2020

DOI: $10.1039 / \mathrm{d} 0 \mathrm{tb} 00021 \mathrm{c}$

rsc.li/materials-b

\section{Influence of molecular weight on transdermal delivery of model macromolecules using hydrogel-forming microneedles: potential to enhance the administration of novel low molecular weight biotherapeutics}

\author{
Aaron R. J. Hutton, Maelíosa T. C. McCrudden, Eneko Larrañeta and \\ Ryan F. Donnelly (D) *
}

With a view to improve the current monoclonal antibody-based therapies dominating the pharmaceutical market, low molecular weight (MW) protein-based macromolecules, such as recombinant antibody fragments, typically within the range of 10-70 kDa, have been developed. Previously, our group successfully delivered Avastin ${ }^{\circledR}$, a monoclonal antibody ( $\mathrm{mAb}$ ) across the skin using hydrogel-forming microneedles (MN). However, it is thought that this delivery system can be further enhanced using novel, lower MW biomolecules. To address this perception, in the current study, FITC-dextran of different MWs $(10,70$ and $150 \mathrm{kDa})$ was used to model the transdermal delivery of low MW biotherapeutics and mAbs with MWs of approximately $150 \mathrm{kDa}$. Conversely, fluorescein sodium was the compound selected to model hydrophilic, low MW drugs. As expected, fluorescein sodium produced the greatest cumulative permeation $(637.4 \pm 42.69 \mu \mathrm{g})$. The amounts of FITC-dextran $10 \mathrm{kDa}$ and $150 \mathrm{kDa}$ which permeated across neonatal porcine skin in vitro were $462.17 \pm 65.85 \mu \mathrm{g}$ and $213.54 \pm 15.19 \mu \mathrm{g}$ after $24 \mathrm{~h}$, respectively. The results collated here suggest that the delivery of emerging novel biotherapeutics, via 'super swelling' hydrogel-forming MNs, have the potential to result in greater permeation across human skin, compared to the delivery of mAbs delivered via the same route.

\section{Introduction}

During the last 30 years, significant research and development funds and resources have focused on antibody-based therapies. ${ }^{1}$ This field of drug treatment, driven by monoclonal antibodies (mAbs), has resulted in biotherapeutic compounds controlling the top 10 globally best-selling drugs. To use one biological drug class as an example, tumour necrosis factor- $\alpha$ (TNF- $\alpha$ ) inhibitors have been proven to effectively induce remission in immunological disorders such as rheumatoid arthritis, Crohn's disease, ulcerative colitis and psoriasis. ${ }^{2-4}$ To this end, the most successful drug on the market is Humira ${ }^{\mathbb{B}}$ (Adalimumab), an anti TNF- $\alpha$ mAb,

School of Pharmacy, Queen's University Belfast, 97 Lisburn Road, Belfast BT9 7BL, UK. E-mail: r.donnelly@qub.ac.uk which provided over $\$ 19$ billion in revenue in $2018 .{ }^{5}$ Evidently, this is a financially lucrative field of development for drug manufacturers and its success has led to the development of improved treatment strategies. One such strategy involves the development of lower molecular weight (MW) protein-based macromolecules. ${ }^{6-10}$ These alternatives, which include smaller recombinant antibody fragments such as Fab and scFv molecules, have been exploited in autoimmune disorders and certain cancers. ${ }^{11}$ By reducing the $\mathrm{MW}$, these proteins are no longer restricted to systemically accessible targets, a considerable limitation with cancer-based mAb therapeutics. This gives these low MW biotherapeutics the ability to access recessed epitopes in cancer tumours. ${ }^{12}$ Furthermore, the reduced complexity of low MW proteins makes their pharmacokinetic properties easier to optimise, in addition to reducing manufacturing costs. ${ }^{11}$

Currently, mAbs are limited to high dose administration using syringe and needles, due to their poor stability and high cost. ${ }^{1,13,14}$ With the potential for needle stick injuries, needle phobia and the necessity for dedicated sharps disposal, there is a need to develop new delivery systems to eliminate these costly obstacles. ${ }^{15}$ A possible alternative are hydrogel-forming microneedles (MN) which consist of drug-free, micron scale polymeric needles situated in perpendicular orientation on a base plate to which a separate drug containing reservoir is attached. Following pain-free application to the skin, these MNs imbibe interstitial skin fluid and swell, creating micro-conduits within the skin layers to permit drug delivery into the dermal micro-circulation. ${ }^{16}$ After application, needles become soft and are incapable of reinsertion. This is a significant advantage as it prevents needle stick injury and removes the need for dedicated sharps disposal.

Previously, Singh et al. (2010) modelled the delivery of small, hydrophilic molecules ( $<500 \mathrm{Da}$ ) using hydrogel-forming MNs. This study, which proved that hydrogel-forming MNs enhanced the transdermal delivery of small molecules, subsequently led to the successful delivery of a number of low MW therapeutics across the skin, including theophylline, metronidazole and ibuprofen-sodium. ${ }^{17-19}$ Surprisingly however, despite the emergence 
of biotherapeutics and the advantages offered by hydrogel-forming MNs, there has been very little research carried out to assess the capabilities of this delivery system to enhance the delivery of such molecules. Courtenay et al. (2018) were the first to report the transdermal delivery of a therapeutic antibody using hydrogel-forming MNs. Although Avastin ${ }^{\mathbb{B}}$ (150 kDa) was successfully stabilised in a lyophilised wafer and delivered across neonatal porcine skin in vitro, due to its high MW, a large volume of drug was trapped within the cross-linked network of the MN array. ${ }^{20}$ Furthermore, when applied in vivo, Avastin ${ }^{\circledR}$ was found to drain into the lymphatic system..$^{20}$ The authors of this paper suggested that hydrogel-forming MNs could therefore be used to target cancer metastases within the lymphatic system. To develop this strategy further in a bid to enhance the systemic delivery of biomolecules using hydrogel-forming MNs, it seems logical to assess the capabilities of this delivery system using novel low MW biotherapeutics. By using FITC-dextran compounds of varying MWs as model compounds, the work presented here mimics the transdermal delivery of low and high MW biomolecules, via hydrogel-forming MNs. Specifically, FITC-dextran $10 \mathrm{kDa}$ was chosen to model an unaltered form of a low MW biotherapeutic. FITC-dextran $70 \mathrm{kDa}$ was chosen to model the half-life extended alternative of these compounds, with FITC-dextran $150 \mathrm{kDa}$ modelling mAbs. As Singh et al. (2010) proved that low MW drugs can be successfully delivered across the skin using hydrogel-forming MNs, fluorescein sodium (376 Da) was utilised in this work as a model hydrophilic drug. Therefore, leading on from the original studies carried out in our research group and outlining the delivery capabilities of hydrogel-forming MNs, this work was designed with a view to informing upon the potential enhanced permeation of novel low MW biotherapeutics when compared to mAbs tested previously.

\section{Materials and methods}

\section{Chemicals}

Fluorescein isothiocyanate-dextran (FITC-dextran 10, 70 and $150, \mathrm{MW}=8000-12000 \mathrm{Da}, \mathrm{MW}=63000-77000 \mathrm{Da}$ and $\mathrm{MW}=$ 140 000-160 $000 \mathrm{Da}$ ) were purchased from TdB Consultancy AB (Uppsala, Sweden). Fluorescein sodium (MW $=376 \mathrm{Da})$ and poly(ethylene glycol) (PEG) with MW = 10000 Da were purchased from Sigma-Aldrich, Dorset, UK. Gantrez ${ }^{\circledR}$ S-97, a co-polymer of methyl vinyl ether and maleic acid (PMVE/MA) was a gift from Ashland, Kidderminster, UK. Sodium carbonate $\left(\mathrm{Na}_{2} \mathrm{CO}_{3}\right)$ was purchased from BDH Laboratory Supplies, London, UK. Cryogel SG3 was provided by PB Gelatins, Pontypridd, UK and Pearlitol ${ }^{\mathbb{R}}$ 50C-Mannitol was supplied by Roquette, Lestrem, France. All other chemicals were of analytical grade.

\section{Manufacture of hydrogel-forming MNs}

'Super swelling' hydrogel-forming MNs were prepared using a formulation published previously. ${ }^{17}$ Due to the low cross-linked density, this has been described as the optimum hydrogel formulation for rapid drug delivery. ${ }^{17,21}$ In short, approximately $500 \mathrm{mg}$ of an aqueous blend of $20 \% \mathrm{w} / \mathrm{w}$ Gantrez ${ }^{\mathrm{R}} \mathrm{S}-97,7.5 \% \mathrm{w} / \mathrm{w}$

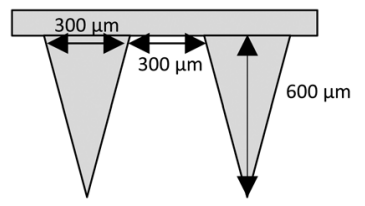

Fig. 1 Schematic representation of a 'super-swellable' hydrogel-forming $\mathrm{MN}$ with conical shaped needles, $600 \mu \mathrm{m}$ in height, interspacing $300 \mu \mathrm{m}$ and base widths of $300 \mu \mathrm{m}$

PEG 10000 and $3 \% \mathrm{w} / \mathrm{w} \mathrm{Na}_{2} \mathrm{CO}_{3}$ was poured into laser engineered silicone moulds, composed of $121(11 \times 11)$ conical holes, $600 \mu \mathrm{m}$ in depth, interspacing $300 \mu \mathrm{m}$ and base widths of $300 \mu \mathrm{m}$ (Fig. 1). Each mould was centrifuged at $3500 \mathrm{rpm}$ for $15 \mathrm{~min}$ and allowed to dry under ambient conditions for $48 \mathrm{~h}$. Following this, MNs were placed in an oven at $80{ }^{\circ} \mathrm{C}$ for $24 \mathrm{~h}$ to facilitate crosslinking. MNs were then demoulded and sidewalls removed using a heated scalpel.

\section{Mechanical testing of hydrogel-forming MNs}

Compression and insertion characteristics of 'super swellable' MNs were assessed using a TA.XT2 Texture Analyser. Before compression, MN heights were measured using a Leica EZ4W stereo microscope. Previous studies have suggested that 8 layers of Parafilm ${ }^{\circledR} \mathrm{M}$ exhibit excellent similarity to porcine skin. ${ }^{21-24}$ Therefore, to artificially simulate $\mathrm{MN}$ insertion in a biological membrane, each MN array was placed above the eight layered polymeric film on the Texture Analyser platform. A previous study using human volunteers showed that when applying MNs, forces were exerted within the range of $0-50 \mathrm{~N}$, with an average maximal force of $30 \mathrm{~N}^{21}$ For this reason, $30 \mathrm{~N}$ was selected as the application force within this study. Taking this into account, a probe was lowered at a speed of $1.19 \mathrm{~mm} \mathrm{~s}^{-1}$ and a force of $30 \mathrm{~N}$, held for $30 \mathrm{~s}$ was applied to each MN array. Following insertion, MNs were removed from the Parafilm ${ }^{\circledR} \mathrm{M}$ and needle height remeasured to determine compression, expressed as percentage height reduction. MN morphology was also visually assessed before and after compression using scanning electron microscopy (SEM) (TM 3030 Tabletop Microscope, Hitachi, Japan). To assess insertion, each Parafilm ${ }^{\circledR}$ M layer was examined under the microscope and the number of holes counted. Insertion in each layer is regarded as successful if the number of holes created in each layer is greater than $20 \% .^{21}$ To confirm insertion into a biological membrane, 'super-swellable' hydrogelforming MNs were placed above full thickness neonatal porcine skin and a $30 \mathrm{~N}$ force was applied for $30 \mathrm{~s}$. Inserted MNs were immediately viewed in situ using an EX1301 optical coherence tomography (OCT) microscope (Michelson Diagnostics, Kent, UK).

\section{Fabrication of FITC-dextran and fluorescein sodium lyophilised wafers}

FITC-dextran $10 \mathrm{kDa}, 70 \mathrm{kDa}, 150 \mathrm{kDa}$ and fluorescein sodium (376 Da) lyophilised wafers were prepared using different combinations of gelatin, mannitol and deionised water (Table 1). To prepare each formulation, the fluorescent compound was first dissolved in deionised water and heated to $37{ }^{\circ} \mathrm{C}$. Mannitol and gelatin were added, followed by hand mixing to facilitate 
Table 1 Different formulations used to produce lyophilised wafers containing FITC-dextran $10 \mathrm{kDa}\left(F_{10}\right), 70 \mathrm{kDa}\left(F_{70}\right), 150 \mathrm{kDa}\left(F_{150}\right)$ and fluorescein sodium $\left(F_{\mathrm{Na}}\right)$

\begin{tabular}{lllll}
\hline ID & $\begin{array}{l}\text { Gelatin } \\
(\% \mathrm{w} / \mathrm{w})\end{array}$ & $\begin{array}{l}\text { Mannitol } \\
(\% \mathrm{w} / \mathrm{w})\end{array}$ & $\begin{array}{l}\text { Analyte } \\
(\% \mathrm{w} / \mathrm{w})\end{array}$ & $\begin{array}{l}\text { Water } \\
(\% \mathrm{w} / \mathrm{w})\end{array}$ \\
\hline $\mathrm{F}_{10} 1$ & 3 & 3 & 0.5 & 93.5 \\
$\mathrm{~F}_{10} 2$ & 5 & 3 & 0.5 & 91.5 \\
$\mathrm{~F}_{10} 3$ & 7 & 3 & 0.5 & 89.5 \\
$\mathrm{~F}_{10} 4$ & 10 & 5 & 0.5 & 84.5 \\
$\mathrm{~F}_{70} 5$ & 10 & 5 & 0.5 & 84.5 \\
$\mathrm{~F}_{150} 6$ & 10 & 5 & 0.5 & 84.5 \\
$\mathrm{~F}_{\mathrm{Na}} 7$ & 10 & 5 & 0.5 & 84.5 \\
\hline
\end{tabular}

dissolution. The formulations were then sonicated at $37{ }^{\circ} \mathrm{C}$ for $60 \mathrm{~min}$ before casting into open-ended cylindrical moulds with diameters of $11.8 \mathrm{~mm}$ and depths of $2 \mathrm{~mm}$. Each formulation was frozen at $-80{ }^{\circ} \mathrm{C}$ for $60 \mathrm{~min}$ and was then placed in a freeze dryer. Using an adapted version of a previously described method, the lyophilisation process involved; primary drying for $90 \mathrm{~min}$ at a shelf temperature of $-40{ }^{\circ} \mathrm{C}, 90 \mathrm{~min}$ at a shelf temperature of $-30{ }^{\circ} \mathrm{C}, 90 \mathrm{~min}$ at a shelf temperature of $-20^{\circ} \mathrm{C}$, $530 \mathrm{~min}$ at a shelf temperature of $-10{ }^{\circ} \mathrm{C}$ and $90 \mathrm{~min}$ at a shelf temperature of $0-10{ }^{\circ} \mathrm{C} .{ }^{17}$ The secondary drying phase was performed over $660 \mathrm{~min}$ at a shelf temperature of $25{ }^{\circ} \mathrm{C}$ and a vacuum pressure of $50 \mathrm{mTorr}$.

\section{Dissolution of FITC-dextran and fluorescein sodium loaded lyophilised wafers}

To determine the dissolution time of each lyophilised wafer, $20 \mathrm{~mL}$ of phosphate buffered saline (PBS) (pH 7.4) solution was added to a glass vial, heated to $37{ }^{\circ} \mathrm{C}$ and stirred at $200 \mathrm{rpm}$. Lyophilised wafers were then added to the PBS (pH 7.4) and the dissolution time was recorded, based on visual inspection.

\section{Determination of FITC-dextran and fluorescein sodium recovery from lyophilised wafers}

FITC-dextran and fluorescein sodium recovery was assessed through a process of dissolution and quantification using fluorescence spectroscopy. Following complete dissolution, samples were removed, diluted appropriately in PBS (pH 7.4) and quantified using fluorescence spectroscopy.

\section{Saturation solubility of FITC-dextran and fluorescein sodium}

To determine saturation solubility, the four compounds were added in excess into separate glass vials containing PBS (pH 7.4) and stirred at $200 \mathrm{rpm}$ for $24 \mathrm{~h}$ at $37^{\circ} \mathrm{C}$. The saturated solution was then filtered using Minisart ${ }^{\mathbb{R}} 0.2 \mu \mathrm{m}$ filters, diluted appropriately and analysed using fluorescence spectroscopy.

\section{In vitro permeation of FITC-dextran and fluorescein sodium using modified Franz cells}

FITC-dextran and fluorescein sodium permeation through dermatomed $(350 \mu \mathrm{m})$ neonatal porcine skin, which was acquired from stillborn piglets, was quantified using modified Franz diffusion cells. Skin integrity was confirmed using transepidermal water loss (TEWL). Each receiver compartment was filled with PBS (pH 7.4), stirred at $600 \mathrm{rpm}$ using a metal stirring bar and left to equilibrate at $37 \pm 1{ }^{\circ} \mathrm{C}$ for $12 \mathrm{~h}$. Following the $12 \mathrm{~h}$ period, skin samples were shaved and fixed to the donor compartment using cyanoacrylate glue. MN arrays were then placed into the centre of each donor compartment and manual thumb pressure was applied for $30 \mathrm{~s}$. An aliquot of water $(20 \mu \mathrm{L})$ was then dispensed onto the $\mathrm{MN}$ baseplate, after which the lyophilised wafer containing the fluorescent compound was placed on top. A $5.0 \mathrm{~g}$ stainless steel weight was placed above the wafer to ensure $\mathrm{MN}$ insertion was maintained throughout the $24 \mathrm{~h}$ period. The donor compartment was then carefully placed on top of the receiver compartment and securely clamped into place. The sampling arm was occluded with Parafilm ${ }^{\mathbb{R}} \mathrm{M}$ to prevent evaporation. Samples $(200 \mu \mathrm{L})$ were removed at specified time points and diluted appropriately in PBS (pH 7.4). An equal volume of fresh pre-warmed PBS (pH 7.4) was added to the receiver vessel to replace this.

\section{Pharmaceutical analysis of FITC-dextran and fluorescein sodium}

Fluorescence analysis was carried out using a BMG FLUOstar Omega Fluorescence Microplate Reader (BMG LabTech, Ortenberg, Germany) at an excitation wavelength $485 \mathrm{~nm}$ and emission wavelength $520 \mathrm{~nm}$. Method validation was performed according to guidelines detailed by the ICH of Technical Requirements for Registration of Pharmaceuticals for Human use Validation of Analytical Procedures Q2 $\left(\mathrm{R}_{1}\right)$ 2005. FITC-dextran stock solutions $\left(3 \mathrm{mg} \mathrm{mL}{ }^{-1}\right.$ ) were prepared in phosphate buffered saline (PBS) (pH 7.4). Working standards of each stock solution were prepared by diluting with appropriate volumes of PBS (pH 7.4). Through a series of serial dilutions, standards were prepared in the range of $0.78 \mu \mathrm{g} \mathrm{mL} L^{-1}$ to $100 \mu \mathrm{g} \mathrm{mL} L^{-1}$. To produce a stock solution of fluorescein sodium $\left(10 \mathrm{mg} \mathrm{mL}{ }^{-1}\right)$, fluorescein sodium was dissolved in PBS (pH 7.4). Through serial dilution, standards were prepared in the range of $0.08 \mu \mathrm{g} \mathrm{mL}^{-1}$ to $2.5 \mu \mathrm{g} \mathrm{mL}{ }^{-1}$. Least squares linear regression analysis and correlation analysis over three consecutive days was used to calculate the coefficient of determination $\left(R^{2}\right)$ and line equation. Limit of detection (LoD) and limit of quantification (LoQ) were calculated using eqn (1) and (2), with $S$ defined as the gradient of the calibration curve and $\sigma$ the standard deviation of the response, determined using the standard error of the $y$-intercepts on the regression line.

$$
\begin{aligned}
& \mathrm{LoD}=\frac{3.3 \sigma}{S} \\
& \mathrm{LoQ}=\frac{10 \sigma}{S}
\end{aligned}
$$

\section{Statistical analysis}

Statistical analysis was performed using GraphPad Prism version 6.07 (GraphPad Software Inc., San Diego, California). This included calculation of means and standard deviations. Paired and unpaired $t$-tests, in addition to Mann Whitney $U$ and Kruskal Wallis tests, were implemented where appropriate, with $p<0.05$ denoting statistical significance. 
a)

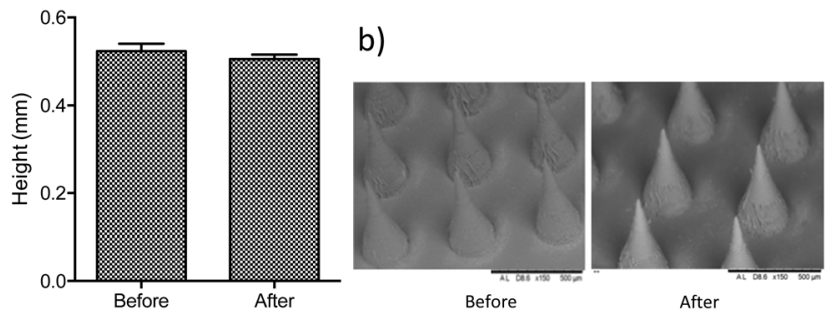

Fig. 2 (a) Compression analysis of $20 \% \mathrm{w} / \mathrm{w}$ Gantrez ${ }^{\circledR} \mathrm{S}-97,7.5 \% \mathrm{w} / \mathrm{w}$ PEG $10000+3 \% \mathrm{w} / \mathrm{w} \mathrm{Na} \mathrm{CO}_{3}$ ('super swelling') MNs. Means \pm S.D. $n=10$. (b) SEM images of a 'super-swelling' $M N$ before and after the application of a $30 \mathrm{~N}$ force for $30 \mathrm{~s}$

\section{Results}

\section{Mechanical testing of 'super swelling' hydrogel-forming MNs}

To investigate the effect of application force on MN compression, needle height was first measured using a Lecia EZ4W stereo microscope. A $30 \mathrm{~N}$ force was then applied to each 'super swellable' hydrogel-forming MN for $30 \mathrm{~s}$ using a TA.XT2 Texture analyser and MN height remeasured as shown in Fig. 2a. Scanning electron microscopy (SEM) showed that there was no visible difference in needle morphology after compression (Fig. 2b).

Before compression, 'super swellable' MNs had mean needle heights of $0.523 \pm 0.017 \mathrm{~mm}$. Following compression, needle heights measured a mean of $0.511 \pm 0.042 \mathrm{~mm}$. There was no significant difference in needle height following the application of the documented compression force ( $p=0.8457)$.

To determine insertion characteristics, 'super swelling' hydrogel-forming MNs were first examined under a Lecia EZ4W stereo microscope to confirm complete needle formation (Fig. 3a and b). Each MN was then placed on 8 layers of Parafilm ${ }^{\circledR} \mathrm{M}$. Following the application of a $30 \mathrm{~N}$ force, needle insertion was visually assessed using an OCT microscope (Fig. 3c). To enable a direct comparison between insertion into an artificial and a biological membrane, 'super-swellable' MNs were placed above full thickness porcine skin and the same force (30 N) was applied for $30 \mathrm{~s}$. Using OCT, it was visually confirmed that this $\mathrm{MN}$ formulation has the strength to insert into both membrane types (Fig. 3c and d). To measure the depth of insertion, the number of holes in each Parafilm ${ }^{\circledR}$ M layer were counted using a Lecia EZ4W stereo microscope. As shown in Fig. 3e, more than $20 \%$ of the needles penetrated through the third layer of Parafilm ${ }^{\circledR} \mathrm{M}$ and less than $20 \%$ through the fourth layer of Parafilm ${ }^{\circledR}$ M. For penetration to be regarded as successful, there must be greater than $20 \%$ needle penetration into a specific layer of the Parafilm ${ }^{\circledR}$ M. $^{21}$ Therefore, as each layer is $126 \mu \mathrm{m}$ thick, the insertion depth was in the range of 378-504 $\mu \mathrm{m}$.

\section{Fabrication of lyophilised wafers containing FITC-dextran and fluorescein sodium}

Using FITC-dextran $10 \mathrm{kDa}$, four different gelatin to mannitol ratios were used in the production of a range of lyophilised wafers, using an iterative approach (Table 1). Each formulation was poured into $250 \mathrm{mg}$ open-ended cylindrical moulds, frozen at $-80{ }^{\circ} \mathrm{C}$ for $1 \mathrm{~h}$ and then freeze dried over a $25 \mathrm{~h}$ cycle. a) $1 \mathrm{~mm}$

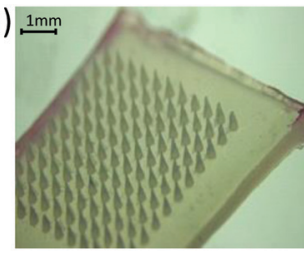

c)

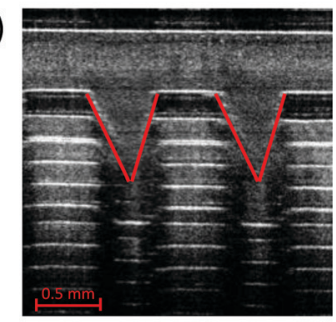

b)

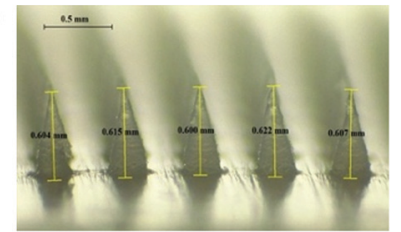

d)

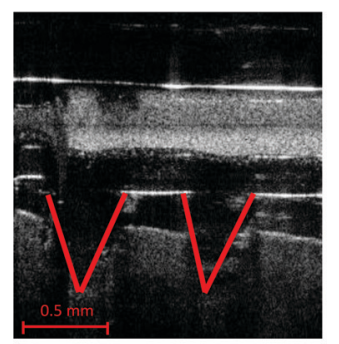

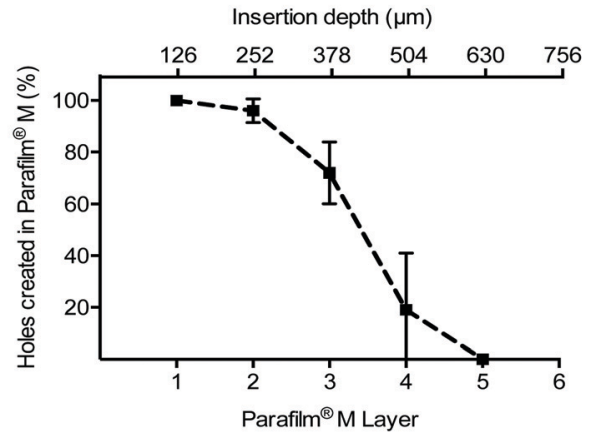

Fig. 3 (a) Light microscope image of a $20 \%$ w/w Gantrez ${ }^{\circledR}$ S- $97,10 \%$ w/w PEG $10000+3 \% \mathrm{w} / \mathrm{w} \mathrm{Na} \mathrm{CO}_{3}$ (super swelling) hydrogel-forming $\mathrm{MN}$. (b) Light microscope image confirming the heights $(600 \mu \mathrm{m})$ of a 'super swelling' hydrogel-forming MN. (c) Optical coherence tomography (OCT) image showing the insertion of a 'super swelling' hydrogel-forming $M N$ into 8 layers of Parafilm ${ }^{\mathbb{R}} \mathrm{M}$ following the application of a $30 \mathrm{~N}$ force for $30 \mathrm{~s}$. (d) OCT image showing the insertion of a 'super swelling' hydrogel-forming $\mathrm{MN}$ into full thickness porcine skin following the application of a $30 \mathrm{~N}$ force for 30 s. (e) Graphical representation of the number of Parafilm ${ }^{\circledR} M$ layers penetrated, and percentage holes created within each layer following a $30 \mathrm{~N}$ force applied for $30 \mathrm{~s}$ to 'super swelling' hydrogel-forming MNs. Means \pm S.D., $n=3$.

As shown in Fig. 4, $\mathrm{F}_{10} 1$ and $\mathrm{F}_{10} 2$ were homogenous but possessed poor structural integrity, both crumbling during demoulding. $\mathrm{F}_{10} 3$ and $\mathrm{F}_{10} 4$ were homogenous and displayed good structural integrity. Consequently, the gelatin to mannitol ratio used to produce $\mathrm{F}_{10} 4$ was tested with the remaining three analytes. All three other formulations $\left(\mathrm{F}_{70} 5, \mathrm{~F}_{150} 6\right.$ and $\left.\mathrm{F}_{\mathrm{Na}} 7\right)$ produced homogenous, intact wafers.

\section{Pharmaceutical analysis of FITC-dextran and fluorescein sodium}

Quantification of FITC-dextran and fluorescein sodium was determined using fluorescence spectroscopy $\left(\lambda_{\mathrm{ex}}=480 \mathrm{~nm}\right.$ and $\left.\lambda_{\mathrm{em}}=520 \mathrm{~nm}\right)$. Calibration curves were run in triplicate over a three-day period to adhere to ICH standards. The limits of detection (LoD) and quantification (LoQ) are presented in Table 2.

\section{FITC-dextran and fluorescein sodium dissolution and recovery}

As $\mathrm{F}_{10} 1$ and $\mathrm{F}_{10} 2$ wafers displayed poor structural integrity, they were not considered for further studies. When placed into $20 \mathrm{~mL}$ of PBS ( $\mathrm{pH} 7.4), \mathrm{F}_{10} 3$ and $\mathrm{F}_{\mathrm{Na}} 7$ underwent complete dissolution after $8 \mathrm{~min}$. $\mathrm{F}_{150} 6$ had a dissolution time of $6 \mathrm{~min} 30 \mathrm{~s}$, whereas $\mathrm{F}_{10} 4$ and $\mathrm{F}_{70} 5$ 

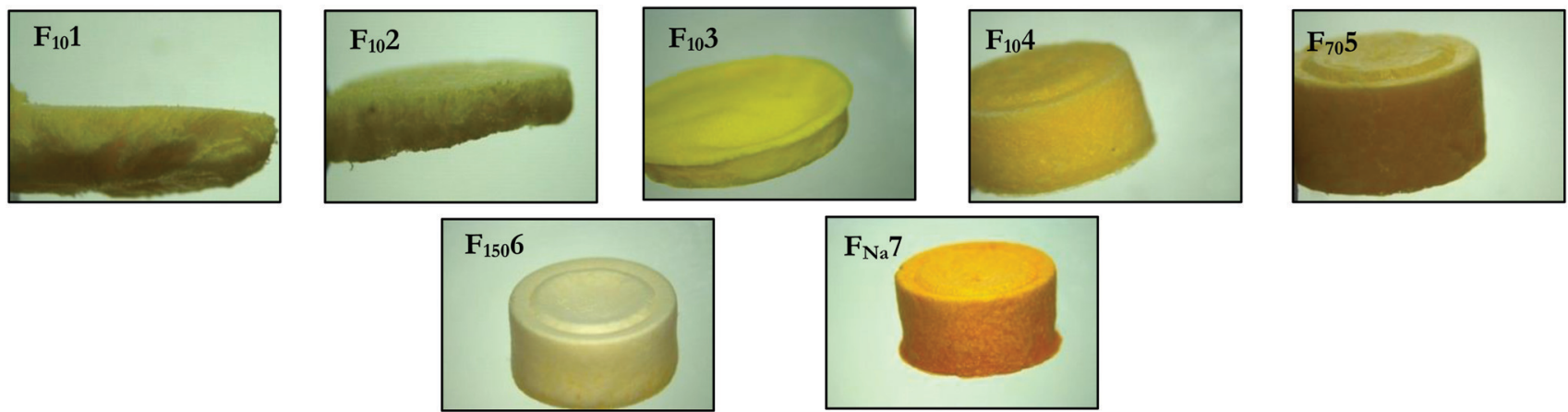

Fig. 4 Light microscope images of FITC-dextran 10 kDa, 70 kDa, 150 kDa and fluorescein sodium lyophilised wafers as detailed in Table 1.

Table 2 Calibration parameters for FITC-dextran $10 \mathrm{kDa}, 70 \mathrm{kDa}, 150 \mathrm{kDa}$ and fluorescein sodium using fluorescence spectroscopy, as represented by coefficient of determination $\left(R^{2}\right)$, limit of detection and limit of quantification

\begin{tabular}{llllll}
\hline Analyte & Concentration range $\left(\mu \mathrm{g} \mathrm{mL} \mathrm{mL}^{-1}\right)$ & $R^{2}$ & Equation of regression line & LoD $\left(\mu \mathrm{g} \mathrm{mL} \mathrm{mL}^{-1}\right)$ & LoQ $\left(\mu \mathrm{g} \mathrm{mL} \mathrm{m}^{-1}\right)$ \\
\hline FITC-dextran 10 kDa & $0.78-100$ & 0.997 & $y=1.4489 x-0.632$ & 0.95 & 2.87 \\
FITC-dextran 70 kDa & $0.78-100$ & 0.999 & $y=1.0224 x-0.6506$ & 1.61 & 4.88 \\
FITC-dextran 150 kDa & $0.78-100$ & 0.997 & $y=1.3352 x-1.5646$ & 1.00 & 3.02 \\
Fluorescein sodium & $0.08-2.50$ & 0.998 & $y=41.54 x+1.5603$ & 0.09 & 0.28 \\
\hline
\end{tabular}

had dissolution times of $5 \mathrm{~min}$. To determine the percentage analyte recovery, $\mathrm{F}_{10} 3, \mathrm{~F}_{10} 4, \mathrm{~F}_{70} 5, \mathrm{~F}_{150} 6$ and $\mathrm{F}_{\mathrm{Na}} 7$ solutions were diluted and quantified using fluorescence spectroscopy. The dissolution of $\mathrm{F}_{10} 3$, $\mathrm{F}_{10} 4 \mathrm{~F}_{70} 5, \mathrm{~F}_{150} 6 \& \mathrm{~F}_{\mathrm{Na}} 7$ resulted in percentage recoveries of $80 \pm$ $6.9 \% ; 95.9 \pm 4.0 \% ; 93.0 \pm 0.60 \% ; 95.7 \pm 5.4 \%$ and $96.0 \pm 5.7 \%$ respectively. Assessing structural integrity, dissolution time and percentage recovery, $\mathrm{F}_{10} 3$ was excluded from in vitro studies due a slow dissolution time and low analyte recovery.

\section{Saturation solubility of FITC-dextran and fluorescein sodium}

The saturation solubility of each fluorescent compound was calculated in PBS (pH 7.4) to ensure sink conditions were not exceeded (Table 3). Maintaining sink conditions in vitro is important in ensuring the rate of diffusion is not affected throughout the study. Fluorescein sodium, with a saturation solubility value of $466 \mathrm{mg} \mathrm{mL}^{-1}$, displayed the greatest solubility. Defining sink conditions as 10 times the volume needed to completely solubilise the analyte, it is apparent that the concentrations of all four analytes in vitro did not exceed sink conditions. ${ }^{25}$

FITC-dextran and fluorescein sodium in vitro permeation study FITC-dextran permeation through porcine skin in vitro using 'super-swelling' hydrogel-forming MNs was quantified using Franz diffusion cells over $24 \mathrm{~h}$ (Fig. $5 \mathrm{a}$ and b).

Table 3 Saturation solubility concentrations of FITC-dextran $10 \mathrm{kDa}$, $70 \mathrm{kDa}, 150 \mathrm{kDa}$ and fluorescein sodium in PBS $(\mathrm{pH} 7.4)$, stirred at

$200 \mathrm{rpm}$ at $37^{\circ} \mathrm{C}$. Means \pm S.D. $n=3$

\begin{tabular}{lc}
\hline Analyte & $\begin{array}{l}\text { Mean saturation concentration } \pm \text { S.D. } \\
\left(\mathrm{mg} \mathrm{mL}^{-1}\right)\end{array}$ \\
\hline FITC-dextran $10 \mathrm{kDa}$ & $91.93 \pm 4.22$ \\
FITC-dextran $70 \mathrm{kDa}$ & $88.13 \pm 3.26$ \\
FITC-dextran $150 \mathrm{kDa}$ & $61.76 \pm 0.86$ \\
Fluorescein sodium & $466 \pm 51.61$
\end{tabular}

Between 0-6 h, there was no significant difference in cumulative permeation between all four fluorescent compounds $(p=0.1242)$. However, over a $24 \mathrm{~h}$ period, fluorescein sodium produced the greatest cumulative permeation, equivalent to $637.4 \pm 42.69 \mu \mathrm{g}$ $(46.55 \pm 4.67 \%)$. FITC-dextran $10 \mathrm{kDa}$ produced a cumulative permeation of $462.17 \pm 65.85 \mu \mathrm{g}$, equivalent to $35.28 \pm 3.71 \%$ of the incorporated fluorescent compound over $24 \mathrm{~h}$. Comparing both compounds, the cumulative permeation of fluorescein sodium and FITC-dextran $10 \mathrm{kDa}$ was deemed to be statistically significant $(p=0.0011)$. With reference to FITC-dextran $10 \mathrm{kDa}$, FITC-dextran $150 \mathrm{kDa}$ resulted in a significantly lower permeation over $24 \mathrm{~h}$ $(213.54 \pm 15.19 \mu \mathrm{g})$, equivalent to only $12.14 \pm 2.73 \%$ release, ( $p=0.0079)$. Interestingly, FITC-dextran $150 \mathrm{kDa}$ displayed a similar permeation profile to FITC-dextran $70 \mathrm{kDa}$. In addition, there was no statistical difference in percentage permeation after $24 \mathrm{~h}$ between FITC-dextran $70 \mathrm{kDa}$ and $150 \mathrm{kDa}(p=0.1349)$.

\section{Discussion}

High MW biomolecules, particularly mAbs $(\sim 150 \mathrm{kDa})$, are now the most successful drug class on the market, with six of the top 10 best selling drugs derived from mAbs. ${ }^{5}$ Despite this success, mAbs are typically administered using a needle and syringe. Although needle and syringe administration is one of the most commonly used methods of drug delivery, fear of needles can result in therapy refusal. A meta-analysis report on needle phobia by McLenon et al. (2019), found that needle phobia in adolescents ranged from $20-50 \%$ and $20-30 \%$ in young adults. ${ }^{26}$ Another issue with this form of administration is that non-compliance is a key contributor in needle stick injuries. Alarmingly, between 2012-2017, the number of successful claims for needle stick injuries was 1213, costing the National Health Service (NHS) in excess of $£ 4$ million. ${ }^{27}$ Therefore, there is 

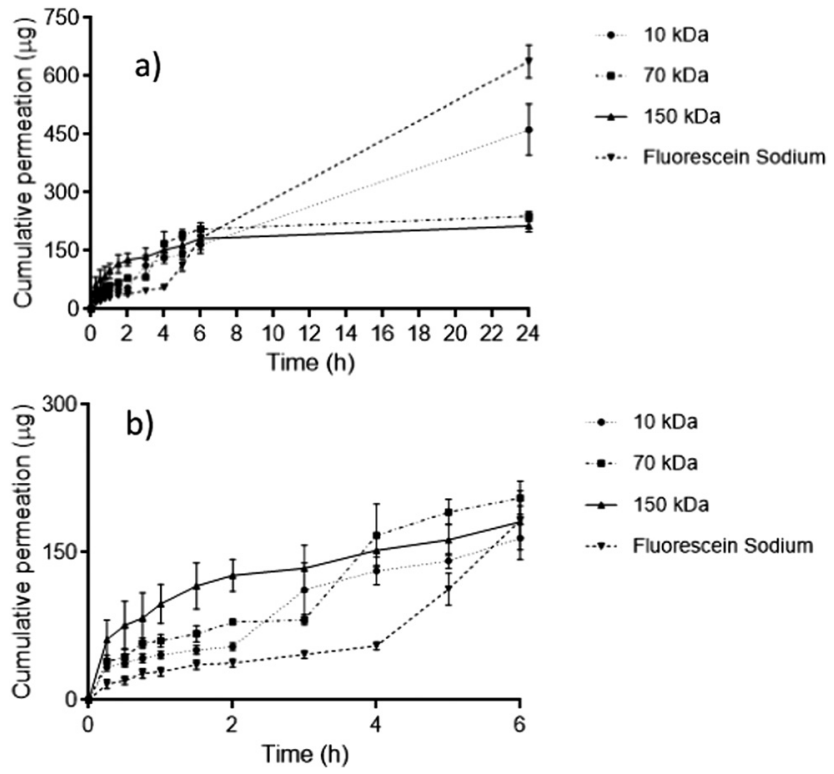

Fig. 5 (a) In vitro permeation profiles of FITC-dextran $10 \mathrm{kDa}, 70 \mathrm{kDa}$, $150 \mathrm{kDa}$ and fluorescein sodium through dermatomed $(350 \mu \mathrm{m})$ neonatal porcine skin over $24 \mathrm{~h}$ using 'super swellable' hydrogel-forming MNs. Means \pm S.D., $n=5$. (b) In vitro permeation profiles between $0-6 \mathrm{~h}$ within the same study.

a need to develop alternative methods of administration to help alleviate these issues.

Microneedles have the potential to offer a viable alternative. The field of microneedle (MN) drug delivery has expanded considerably in the last decade, with advances in technology enabling several types of MNs to be exploited. The first modalities developed were solid and hollow MNs, fabricated from silicon, metals and glass. ${ }^{28-32}$ In particular, hollow MNs have been tested previously in the delivery of biologics. Harvey et al. (2011) used a delivery device consisting of three $1 \mathrm{~mm} 34 \mathrm{G}$ steel needles incorporated into a fluid-disrupting hub to deliver etanercept (132 kDa) intradermally. This method was compared with intravenous (IV) and subcutaneous (SC) injections. As expected, the IV injection resulted in immediate delivery to the peripheral vasculature. The authors reported that intradermal (ID) administration resulted in rapid uptake into the lymphatic system, a feature not observed with SC delivery. ${ }^{33}$ It was concluded that MN facilitated drug delivery would be valuable in direct lymphatic targeting, with particular benefits in cancer therapy and diagnostic imaging.

Dissolving MNs have been tested with therapeutics possessing a wide range of solubilities and MWs. ${ }^{15,34-37}$ This delivery system has shown to be of considerable interest in bolus dosing due to the short dissolution time of the needle tips. In particular, corium have manufactured a drug-in tip dissolving $\mathrm{MN}$ containing teriparatide, a recombinant human parathyroid hormone indicated in osteoporosis which has successfully completed Phase 2 a clinical trials. ${ }^{38}$ At present, this treatment regime consists of a low dose (20 micrograms) once daily SC injection. Therefore, despite the limited drug loading associated with dissolving MNs, this particular delivery system has shown considerable promise.
Unfortunately, therapeutic antibodies require sustained releases of higher doses to exert their therapeutic effect. Therefore, as drug loading is no longer restricted to what can be loaded into the $\mathrm{MN}$, hydrogel-forming MNs offer a viable delivery option. Our group has tested this alternative method of delivery with Avastin ${ }^{\circledR}$, a mAb, however, the high MW of this biotherapeutic caused entrapment within the cross-linked network of the MN resulting in sub-therapeutic dosing. ${ }^{20}$ Additionally, the large MW of this protein resulted in drainage into the lymphatic system following ID administration, leading to low concentrations in the systemic circulation. $^{20,33}$ To this end, it is envisaged that low MW antibodybased alternatives could overcome these potential delivery issues.

In this study, 'super-swellable' hydrogel-forming MNs were used to facilitate delivery of FITC-dextran of three different MWs and fluorescein sodium in vitro. The experiments were designed to mimic the delivery of novel low MW biomolecules to allow for a direct comparison with mAbs tested previously. Currently, there are five different types of MNs, the most recent addition being hydrogel-forming MNs. These MNs have been shown to be safe to use. Following repeat MN application, results have shown that skin barrier function is not compromised, hereby minimising the possibility of inflammation or infection. Another advantage is that as drugs are not incorporated into or onto the MN, delivery is no longer limited to the loading capacity of the array, a considerable drawback associated with dissolving and coated MNs. Accordingly, hydrogel-forming MNs are of particular interest in the delivery of high dose macromolecules. Although they have been tested using a number of low MW compounds, each with different physical and chemical properties, to date there has been very little work carried out utilising higher MW compounds.

To begin, four FITC-dextran $10 \mathrm{kDa}$ formulations, consisting of different gelatin and mannitol ratios were fabricated into lyophilised wafers. Following a $25 \mathrm{~h}$ freeze drying cycle, $\mathrm{F}_{10} 4$ produced a homogenous wafer with good structural integrity. This was considered a suitable formulation to develop the remaining lyophilised wafers containing FITC-dextran $70 \mathrm{kDa}$, $150 \mathrm{kDa}$ and fluorescein sodium. Similar to FITC-dextran $10 \mathrm{kDa}$, the three other formulations (FITC-dextran $70 \mathrm{kDa}$, $150 \mathrm{kDa}$ and fluorescein sodium) had short dissolution times and high percentage recoveries. Currently, there are no regulated dissolution tests with hydrogel-forming MNs, however, within this study, a suitable dissolution time for each lyophilised wafer was considered to be $<10 \mathrm{~min}$. Nevertheless, it is important that there is a trade-off between dissolution time and structural integrity. For this reason, the wafers chosen for further in vitro testing were homogenous, had good structural integrity and an appropriate dissolution time.

After the successful incorporation of all four compounds into separate lyophilised wafers, in vitro permeation through porcine skin using 'super-swellable' hydrogel-forming MNs was assessed. As the name suggests, a 'super-swelling' hydrogelforming $\mathrm{MN}$ results in a significantly greater swelling capacity compared to hydrogel formulations reported previously by our group. ${ }^{17,19,39}$ This in turn causes the creation of larger microconduits within the skin. ${ }^{17}$ Additionally, the lower density and greater space within the polymer itself, permits the permeation 
of higher MW FITC-dextrans from their hygroscopic wafers, through the MN and into the receiver vessel of the Franz cell. It was interesting to note that there was no significant difference in permeation between all four compounds during 0-6 h period. During this period, the MN itself is imbibing the receiver fluid and swelling, before reaching its maximum swelling capacity at $6 \mathrm{~h}$. After this period, it was observed that increasing the MW of the fluorescein-based compounds decreased the permeability across porcine skin between $6-24 \mathrm{~h}$ period in vitro. A plausible reason for this could be due to FITC-dextran $70 \mathrm{kDa}$ and $150 \mathrm{kDa}$ becoming trapped within the cross-linked network of the hydrogel, as observed with Avastin ${ }^{\circledR}$ which has been tested previously. $^{20}$

An interesting study by Ambati et al. (2000) assessed the in vitro permeability of FITC-dextrans and FITC-IgG in the range of 4-150 kDa across rabbit sclera. The authors found that the sclera was more permeable to globular proteins than to linear dextrans of similar molecular weight. ${ }^{40}$ In this case, hydrodynamic radius was a better predictor of scleral permeability than MW. Considering this phenomenon, in relation to transdermal drug delivery using hydrogel-forming MNs, a comparison can be drawn between the FITC-dextran $150 \mathrm{kDa}$ used in this report and Avastin ${ }^{\circledR}$ used previously. ${ }^{20}$ Although different compositions of mannitol and gelatin were used to yield homogeneous, intact structures, FITC-dextran and Avastin ${ }^{\circledR}$ wafers displayed similar percentage recoveries and dissolution times. Avastin ${ }^{\circledR}$, a globular protein has a hydrodynamic radius of $46 \AA^{41}$ Although FITC-dextran $150 \mathrm{kDa}$ has the same MW, it has a radius of $85 \AA$ due to its linear structure. ${ }^{42}$ Comparing both compounds, the permeation of Avastin ${ }^{\circledR}$ across porcine skin in vitro, was approximately two-fold greater than FITC-dextran $150 \mathrm{kDa}$. This difference is consistent with that found by Ambati et al. (2000), therefore, it is possible that the use of FITC-dextran $10 \mathrm{kDa}$ in this paper could lead to an underestimation in the permeability of low MW protein-based therapeutics across porcine skin in vitro. For this reason, hydrodynamic radius may be a better predictor of transdermal delivery of model compounds using MNs and will form the basis for future studies. Nevertheless, the results obtained within this study certainly suggest that the incorporation of low MW biotherapeutics will result in greater skin permeation when compared to the current mAb based therapeutics. ${ }^{43}$ As such, this study provides a basis and proof of concept for the incorporation of novel low MW proteinbased therapeutics into lyophilised wafers for transdermal delivery using hydrogel-forming MNs.

\section{Conclusions}

Advances in antibody-based engineering have led to the production of low MW biotherapeutics. As these novel proteins are cheaper to manufacture and are not restricted to systemically accessible targets, they are now beginning to be considered as potential alternatives to mAbs in autoimmune and cancerbased therapies. At present, most protein-based therapeutics are administered using a syringe and needle, but it is thought that MNs can offer a viable alternative means of delivery. Previously, Avastin ${ }^{\circledR}$, a mAb, was successfully delivered across porcine skin using hydrogel-forming MNs, however, lower MW biomolecules are thought to further improve transdermal delivery to anatomical sites. Consequently, this study aimed to model the skin permeation of low and high MW biotherapeutics using hydrogel-forming MNs as the delivery vehicle. To achieve this, FITC-dextran of different MWs and fluorescein sodium were delivered across neonatal porcine skin in vitro using hydrogelforming MNs. The amount of FITC-dextran $10 \mathrm{kDa}$ that permeated across the skin membrane was significantly greater than that achieved for FITC-dextran $150 \mathrm{kDa}$. Therefore, this model study has shown that low MW biomolecules will permeate through porcine skin in greater amounts than mAbs tested previously using MNs.

\section{Author contributions}

A. R. J. H. and R. F. D. contributed to the conceptualization of this study. A. R. J. H. contributed to the methodology, validation, formal analysis, investigation, data curation and writing-original draft preparation. E. L., R. F. D. contributed to the resources. M. T. C. M., E. L., R. F. D. contributed to the writing-review and editing. M. T. C. M. and R. F. D. contributed to the supervision, project administration and funding acquisition.

\section{Conflicts of interest}

The authors declare no conflict of interest.

\section{Acknowledgements}

This work was supported in part by the Wellcome Trust (WT094085MA).

\section{Notes and references}

1 J. K. H. Liu, Ann. Med. Surg., 2014, 3, 113-116.

2 NICE 2010, available at: https:/www.nice.org.uk/guidance/ ta195, accessed on 15 April 2019.

3 NICE 2015, available at: https:/www.nice.org.uk/guidance/ ta329, accessed on 15 April 2019.

4 NICE 2010, available at: https:/www.nice.org.uk/guidance/ ta199/evidence, accessed on 15 April 2019.

5 A. Philippidis, Genet. Eng. Biotechnol. News, 2019, 39, 16-17.

6 D. L. Ludwig, et al., J. Biol. Chem., 2005, 280, 19665-19672.

7 B. Schlereth, et al., Cancer Res., 2005, 65, 2882-2889.

8 X. Jimenez, et al., Mol. Cancer Ther., 2005, 4, 427-434.

9 J. S. Haas, Ann. Intern. Med., 2005, 142, 891.

10 P. Å. Nygren, FEBS J., 2008, 275, 2668-2676.

11 P. Holliger and P. J. Hudson, Nat. Biotechnol., 2005, 23, 1126-1136.

12 A. Thakur, M. Huang and L. G. Lum, Blood Rev., 2018, 32, 339-347.

13 P. Chames, M. Van Regenmortel, E. Weiss and D. T. Baty, Br. J. Pharmacol., 2009, 157, 220-233. 
14 R. Rouet, D. Lowe and D. Christ, FEBS Lett., 2014, 588, 269-277. 15 A. R. J. Hutton, et al., Int. J. Pharm., 2018, 541, 56-63.

16 R. F. Donnelly, T. R. Raj Singh and D. A. M. Woolfson, Drug Delivery, 2010, 17, 187-207.

17 R. F. Donnelly, et al., PLoS One, 2014, 9, 1-12.

18 R. F. Donnelly, et al., Adv. Funct. Mater., 2012, 22, 4879-4890.

19 T. R. Raj Singh, D. A. Woolfson and R. F. Donnelly, J. Pharm. Pharmacol., 2010, 62, 829-837.

20 A. J. Courtenay, M. T. C. McCrudden, K. J. McAvoy, H. O. McCarthy and R. Donnelly, Mol. Pharmaceutics, 2018, 15, 3545-3556.

21 E. Larrañeta, et al., Int. J. Pharm., 2014, 472, 65-73.

22 M. S. Lhernould, M. Deleers and A. Delchambre, Int. J. Pharm., 2015, 480, 8-15.

23 S. N. Economidou, et al., Mater. Sci. Eng., C, 2019, 102, 743-755.

24 E. Larrañeta, et al., Int. J. Pharm., 2016, 497, 62-69.

25 B. R. Rohrs, Dissolution Technol., 2001, 8, 6-12.

26 J. McLenon and M. A. M. Rogers, J. Adv. Nurs., 2019, 75, 30-42.

27 NHS Resolution, 2017, available at: https://www.resolution. nhs.uk, accessed on 19 June 2019.

28 P. Khanna, K. Luongo, J. A. Strom and S. Bhansali, J. Micromech. Microeng., 2010, 20, 1-8.
29 P. Khanna, K. Luongo, J. A. Strom and S. Bhansali, Microsyst. Technol., 2010, 16, 973-978.

30 C. O'Mahony, Biomed. Microdevices, 2014, 16, 333-343.

31 F. J. Verbaan, et al., J. Controlled Release, 2007, 117, 238-245.

32 P. M. Wang, M. Cornwell, J. Hill and M. Prausnitz, J. Invest. Dermatol., 2006, 126, 1080-1087.

33 A. J. Harvey, et al., Pharm. Res., 2011, 28, 107-116.

34 J.-Y. Kim, et al., Eur. J. Pharm. Biopharm., 2016, 105, 148-155.

35 J. Mönkäre, et al., J. Controlled Release, 2015, 218, 53-62.

36 K. Lee, C. Y. Lee and H. Jung, Biomaterials, 2011, 32, 3134-3140.

37 H. L. Quinn, L. Bonham, C. M. Hughes and R. Donnelly, J. Pharm. Sci., 2015, 104, 3490-3500.

38 A. C. Anselmo, Y. Gokarn and S. Mitragotri, Nat. Rev. Drug Discovery, 2018, 18, 19-40.

39 T. R. Raj Singh, P. A. McCarron, A. D. Woolfson and R. F. Donnelly, Eur. Polym. J., 2009, 45, 1239-1249.

40 J. Ambati, et al., Invest. Ophthalmol. Visual Sci., 2000, 41, 1181-1185.

41 L. M. Hirvonen, et al., Pharm. Res., 2016, 33, 2025-2032.

42 A. Ekani-Nkodo and D. K. Fygenson, Phys. Rev., 2003, 67, 1-7.

43 K. T. Xenaki, S. Oliveira and P. M. van Bergen en Henegouwen, Front. Immunol., 2017, 8, 1-6. 